\title{
Wine, alcohol and atherosclerosis: clinical evidences and mechanisms
}

P.L. da Luz and S.R. Coimbra
Unidade de Aterosclerose, Instituto do Coração, Hospital das Clínicas, Universidade de São Paulo, São Paulo, SP, Brasil

\section{Correspondence \\ P.L. da Luz \\ Unidade de Aterosclerose \\ Instituto do Coração (InCor) \\ Av. Dr. Eneas C. Aguiar, 44 \\ 05403-000 São Paulo, SP \\ Brasil \\ Fax: +55-11-3069-5447 \\ E-mail: daluzp@incor.usp.br \\ Research supported by FAPESP (No. 1998/10576-5) and Fundação Zerbini.}

Received February 27, 2004

Accepted May 18, 2004

\begin{abstract}
Atherosclerosis is a chronic inflammatory disease which may cause obstructions of the coronary, cerebral and peripheral arteries. It is typically multifactorial, most often dependent on risk factors such as hypercholesterolemia, diabetes, smoking, hypertension, sedentarism, and obesity. It is the single main cause of death in most developed countries due to myocardial infarction, angina, sudden death, and heart failure. Several epidemiological studies suggest that moderate alcohol intake, especially red wine, decrease cardiac mortality due to atherosclerosis. The alcohol effect is described by a J curve, suggesting that moderate drinkers may benefit while abstainers and heavy drinkers are at higher risk. Experimental studies indicate that most beneficial effects of drinking are attributable to flavonoids that are present in red wine, purple grape juice and several fruits and vegetables. The mechanisms include antiplatelet actions, increases in high-density lipoprotein, antioxidation, reduced endothelin-1 production, and increased endothelial nitric oxide synthase expression which causes augmented nitric oxide production by endothelial cells. These findings lead to the concept that moderate red wine drinking, in the absence of contraindications, may be beneficial to patients who are at risk of atherosclerotic cardiovascular events. Moreover, a diet based on fruits and vegetables containing flavonoids may be even more beneficial.
\end{abstract}

\section{Introduction}

Atherosclerosis manifests itself as coronary artery disease (CAD), stroke or peripheral vascular insufficiency and tends to be a progressive process that begins early in life, probably in childhood. Progression of atherosclerosis may be halted or substantially reduced, and even regression of some lesions may be obtained. Reduction of cardiac events has been demonstrated with intense lipid-lowering therapy in patients who al-
Key words

- Coronary disease

- Beverages

- Drinks and cardiac protection

- Flavonoids

- Wine and grape juice ........................ ready suffered cardiac events as well as in those without previous events. The importance of life style changes has also been emphasized, especially weight loss, smoking cessation, exercise, and an appropriate diet.

Several peculiar aspects are involved in life style issues. One of them is the intake of alcoholic beverages, especially red wine. A critical review of the evidences correlating the intake of alcoholic beverages and atherosclerotic diseases, as well as non-vascular diseases, is presented in this review. 


\section{The nature of the atherosclerosis process}

Atherosclerosis is the result of arterial wall/circulating blood element interactions triggered by environmental factors that influence individual genetic components. Vascular alterations in the coronary bed comprise some key elements such as early endothelial dysfunction, inflammatory cellular infiltration, lipid deposition, vascular wall cell proliferation, lumen obstruction, and thrombotic complications at lesion sites (1).

Endothelial cells exert multiple physiological functions, maintaining the integrity of the vascular wall and representing a permeable barrier through which diffusion and active transport of several substances occur; they constitute a non-thrombotic, nonadherent surface for platelets and leukocytes; act upon vascular tone by producing nitric oxide, prostaglandins and endothelins; they also produce and secrete growth factors and cytokines and maintain basement membrane integrity which gives support to collagen and proteoglycans. The strategic location of the endothelium makes it a true "sensor" for hemodynamic and chemical signals from blood, translating them into messages through intracellular transcription mechanisms; on the other hand the endothelium responds to several stimuli by secreting vasoactive substances. Furthermore, the endothelium possesses considerable regional specialization, which is important for local blood flow regulation.

Alterations in one or more of these functions represent early manifestations of endothelial dysfunction and can start cellular interactions with monocytes, T lymphocytes, platelets, and smooth muscle cells (SMCs) leading to the development of atherosclerotic plaques. Several animal and human studies point to endothelial dysfunction as the initial step of atherosclerosis. The vascular response to injury is of the inflammatory type encompassing interactions of several cell groups such as endothelial cells, monocytes/macrophages, $\mathrm{T}$ lymphocytes, platelets, and SMCs. Initial lesions occur especially at sites where normal blood flow is altered, interfering with shear stress and the normal characteristics of the endothelium and reducing nitric oxide (NO) production. These factors occur typically in arterial bifurcations. The process of atheromatous plaque formation can be described to consist of six essential phases, as follows: 1) endothelial dysfunction; 2) penetration of lowdensity lipoprotein (LDL) particles and circulating leukocytes, specifically $\mathrm{T}$ lymphocytes and monocytes, into the subendothelium; 3) LDL oxidation; 4) foam cell formation; 5) SMC migration and proliferation in the subendothelium with matrix synthesis; 6) structural endothelial lesion followed by platelet deposition and thrombus formation.

Several experimental studies have identified endothelial dysfunction as the initial event in induced hypercholesterolemia which results in increased endothelial permeability to lipoproteins and other plasma components. It was shown in primates treated with a cholesterol-rich diet that monocytes adhere very early on to the normal endothelium, and soon penetrate the subendothelial space through normal endothelial cell junctions. Two weeks after diet initiation it is already possible to find LDL particles in the subendothelial space.

Leukocyte deposition on the endothelium and their penetration into the arterial wall are mediated by adhesion molecules expressed in endothelial as well as circulating cells. Vascular cell adhesion molecules (VCAM), intercellular adhesion molecules (ICAM), E-selectin, and endothelial leukocyte adhesion molecules are among the most important adhesion molecules. It is believed that secretion of adhesion molecules is regulated by cytokines synthesized in small concentrations by the arterial endothelium. Such cytokines include inteleukin-1 (IL-1), IL-4, IL-6, tumor necrosis factor- $\alpha$ (TNF- $\alpha)$, and 
interferon- $\gamma$. In the presence of endothelial dysfunction, their concentration increases thus stimulating adhesion molecule production which favors monocyte recruitment and adhesion to the endothelium.

According to the oxidative theory (2), LDL oxidation is a key event. In the presence of macrophages, endothelial cells or SMCs that produce reactive oxygen species, LDL particles go through progressive oxidation. Initially only the lipid components suffer oxidation.

Lipid hydroperoxides, lysophospholipids, carbonyl compounds as well as other biologically active compounds are found in atheroma. Protein components are also oxidized, become antigenic and elicit T-cell responses, and hence provoke an immune reaction. Oxidized LDL (LDLox) is then recognized by scavenger receptors and CD36 on the macrophage surface. Monocytes penetrate the subendothelial space and differentiate into macrophages; these macrophages incorporate large amounts of LDL particles, thus generating foam cells. More recently a specific receptor for LDLox was identified in endothelial cells. Accumulation of foam cells form fatty streaks, the earliest lesion of atherosclerosis visible to the naked eyes. Monocyte chemotactic protein-1 (MCP-1), a powerful chemotactic agent, plays a fundamental role in monocyte adhesion to and penetration of the arterial wall; a family of lymphocyte chemoattractants also attracts $\mathrm{T}$ lymphocytes to the subendothelium. Monocyte differentiation into macrophages and their proliferation are mainly due to macrophage colony-stimulating factor. LDLox exerts several other important functions besides foam cells formation, including MCP-1 and IL-1 production, reduction of monocyte recirculation which retains monocytes in the subendothelium, and a direct toxic action on the endothelium which leads to its structural damage.

Proliferation and migration of SMCs from the media into the subendothelium is an- other key event. Once in the intima SMCs proliferate, produce cytokines, growth factors and extracellular matrix, which mainly contains collagen and proteoglycans. Recently, bone marrow-derived progenitor cells were found in the neointima of mice subjected to arterial balloon injury; these progenitor cells differentiate into SMCs and contribute to neointima formation. A later event is endothelial erosion induced by LDLox; this leads to platelet microthrombus formation, which also causes the production of derived growth factors. At this stage the endothelium may present extensive erosions. The interaction among platelets, endothelium, SMCs, and macrophages determines the degree of cellular proliferation, extracellular matrix formation and consequently the extent of the mature plaque. A peculiar aspect of endothelium participation in atherosclerosis is its potential for regeneration. It has been shown experimentally that the endothelium is able to regenerate structurally and functionally within four weeks after mechanical injury. Such regeneration is greatly dependent on growth factors produced by SMCs. This phenomenon probably is of clinical relevance since it may prevent repeated vascular events that would occur otherwise during increased sympathetic activity, hypertensive crises or heavy acute smoking.

\section{Nitric oxide, endothelins and extension of atherosclerosis}

Several lines of evidence clearly demonstrate that NO prevents experimental atherosclerosis since it reduces leukocyte adhesion and activation, monocyte migration, and endothelial cell activation by cytokines, and inhibits SMC proliferation and platelet adhesion as well as aggregation. On the other hand, endothelin-1 (ET-1) has opposite effects: it promotes leukocyte adhesion and induces monocyte chemotaxis and SMC proliferation in addition to facilitating LDL up- 
take by endothelial cells. Furthermore, ET-1 is a marker of the extent of human atherosclerosis; thus ET-1 levels are significantly higher in symptomatic patients with vascular disease than in normal individuals; also there is a positive correlation between plasma ET-1 concentration and the number of diseased vessels including carotid, coronary and peripheral arteries. Therefore, the endothelium has been shown to modulate the atherosclerotic process both in experimental and human studies, while NO reduces it and ET-1 tends to accentuate it (3).

\section{Risk factors and intracellular signaling}

The mechanisms by which risk factors influence cells participating in the atherosclerosis process have been better understood by analyzing intracellular signaling. Nuclear factor- $\kappa$ B (NFKB) is one such important system. NFKB belongs to a family of redox-sensitive transcriptional factors present in endothelial cells, macrophages, SMCs, leukocytes, cardiomyocytes, and fibroblasts. NFKB can be activated by TNF- $\alpha$, IL- 1 , hyperglycemia, shear stress, LDLox, and oxidative stress among other factors. This process modifies the expression of genes that code for cytokines, inducible nitric oxide synthase, cyclooxygenase 2 , adhesion molecules, immune receptors, IL-8, acute phase proteins, and matrix metalloproteinases. $\mathrm{NF \kappa B}$ is also fundamental for the SMC proliferation induced by thrombin and TNF- $\alpha$ and for ICAM, VCAM and MCP-1 gene expression.

NFKB may therefore contribute to the initiation and progression of atherosclerosis since most pro-inflammatory genes expressed in endothelial cells in the early lesion phases are NFkB dependent. This concept is supported by the observation that genetically NFKB-deficient mice have fewer fatty streaks when kept on a fat-rich diet than wild controls.

\section{Renin-angiotensin system}

Several investigations now indicate the participation of the angiotensin system in the pathophysiology of atherosclerosis. The angiotensin type-1 receptor can be stimulated by several risk factors, including hypercholesterolemia and estrogen deficiency. One important consequence of such stimulation is induction of oxidative stress which directly affects the functions of monocytes, endothelial cells, SMCs, lipids, and coagulation. The resulting endothelial dysfunction promotes plaque formation as well as its rupture and instability. Both experimental and clinical studies have shown that the evolution of atherosclerosis can be significantly reduced by AT1 receptor blockade with losartan or with angiotensin converting enzyme.

\section{Progression and destabilization of lesions}

Human coronary atherosclerosis is clinically characterized by long silent periods in which plaque formation may be restricted to the vessel wall without significant reduction in lumen size or blood flow. This slow progression eventually leads to vessel lumen obstruction, ischemia and clinical manifestations of angina. Another form of evolution is acute instability of the plaque caused by plaque rupture or erosion. This event is induced by plaque inflammation and is directly caused by thrombotic obstruction. Clinical correlates are acute coronary syndromes, acute myocardial infarction or unstable angina.

\section{Relation to wine and alcohol}

Several lines of evidence indicate that flavonoids may influence many factors that participate in the formation and evolution of atherosclerotic plaques. Epidemiological as well as experimental data point in this direc- 
tion; however, the mechanisms involved in such protective effect have only recently started to be elucidated. For instance, red wine flavonoids can decrease ET- 1 production, block NFKB expression and increase NO secretion by endothelial cells. This evidence will be reviewed below.

\section{Cardiovascular diseases}

In 1819, Samuel Blac observed a high rate of CAD in Irish autopsies and an unexplained low rate of CAD cases in France and Mediterranean countries. Since then this phenomenon has been examined more closely by many investigators.

Epidemiological studies indicate that there is a direct relationship between dietary fat intake and the incidence of deaths due to cardiovascular diseases. However, in France, the prevalence of CAD is lower than in several other European and American countries, although dietary fat intake is about the same. Renaud and de Lorgeril (4) attributed this phenomenon to wine consumption. These observations were called the "French paradox". The French paradox, however, does not establish a cause/effect relationship between alcoholic beverages and CAD. Other components of the diet, smoking habit, genetic factors, stress, and exercise might play a role in this relationship.

These findings are in accordance with a study conducted in 17 countries (5) where wine is regularly consumed, which shows an inverse association between alcohol ingestion and cardiac mortality. The beneficial effect of alcohol seems to be due essentially to wine.

Other studies also evaluated the association between different alcoholic beverages and mortality due to CAD or cerebral accidents in the general population. Gronbaek et al. (6) showed a decreased risk for CAD among wine drinkers in a population of 6051 men and 7234 women, 30-70 years of age, prospectively followed for $10-12$ years. The intake of beer or spirits was not associated with risk reduction. Baseline assessment included alcohol intake, smoking habit, income, education, and body mass index. Decreased risk for CAD was evident in individuals ingesting less than $22 \mathrm{~g} /$ day of alcohol, i.e., two glasses of wine.

These investigators concluded that moderate alcohol intake, especially wine, protected from death due to CAD and cerebrovascular accidents. As to the consumption of beer, 3 to 5 glasses a day also decreased the risk when compared to individuals who did not drink alcohol. In contrast, the consumption of three to five glasses of other distilled alcoholic beverages a day was associated with increased mortality.

Klatsky et al. (7) analyzed a Kaiser-Permanent Questionnaire answered by 100,000 people and noted a lower CAD risk in individuals who preferred wine, although beer also reduced the risk. In the Framingham study there was an inverse relationship between alcohol intake and angina, acute myocardial infarction, sudden death and death by CAD, during a 22-year follow-up.

Several other case-controlled studies have shown the protective effect of moderate alcohol intake. For instance, in 1978 a study was begun in England involving 12,321 British doctors who responded to a questionnaire regarding their alcohol consumption (8) and who were followed prospectively for 13 years. By 1991 about one third had died. For all causes of death combined there was a U-shaped curve with a lower mortality for those who consumed 8-14 units of alcohol/ week (1 to 2 units/day); however, there was a highly significant increase in mortality for men drinking more than 28 units/week (Figure 1). For CAD specifically there was only the suggestion of a U-shaped curve, with the lowest mortality among those who drank 15-21 units/week. The authors concluded that: "... the consumption of an average of one or two units of alcohol a day is associated with significantly lower all cause mor- 
tality than is the consumption of no alcohol, or the consumption of substantial amounts".

Similarly, sudden death was examined in 21,537 US males who participated in the Physicians Health Study (9) over 12 years of follow-up. Men who consumed light to moderate amounts of alcohol, i.e., 2 to 6 drinks/ week in the form of either wine, beer or liquor, had a significantly reduced risk of

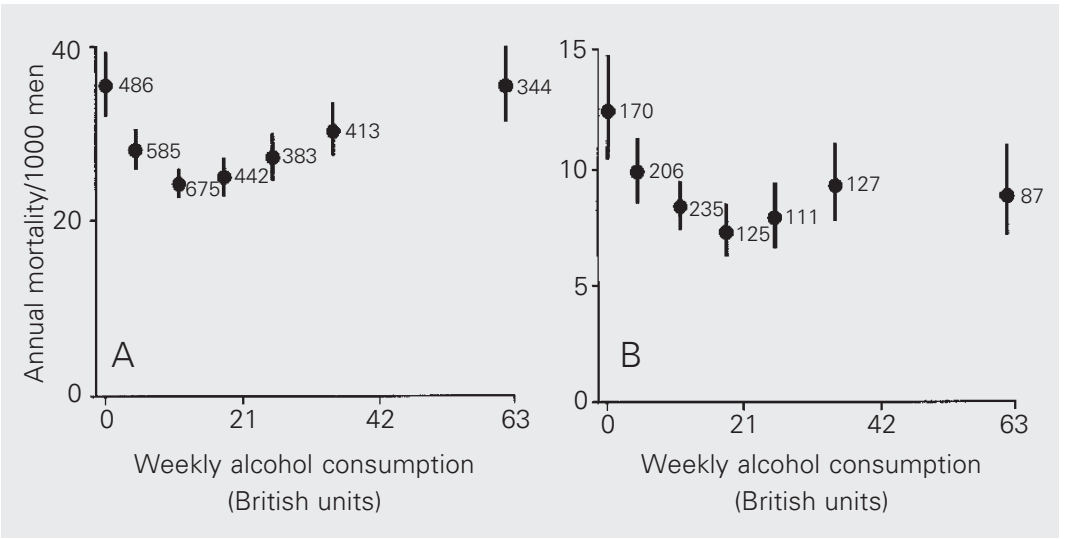

Figure 1. The U-shaped curve for all causes (A) and ischemic heart disease (B) mortality in British doctors in relation to alcohol consumption. British units $=9 \mathrm{~g}$ alcohol (or equivalent). Modified from Ref. 8, with permission.

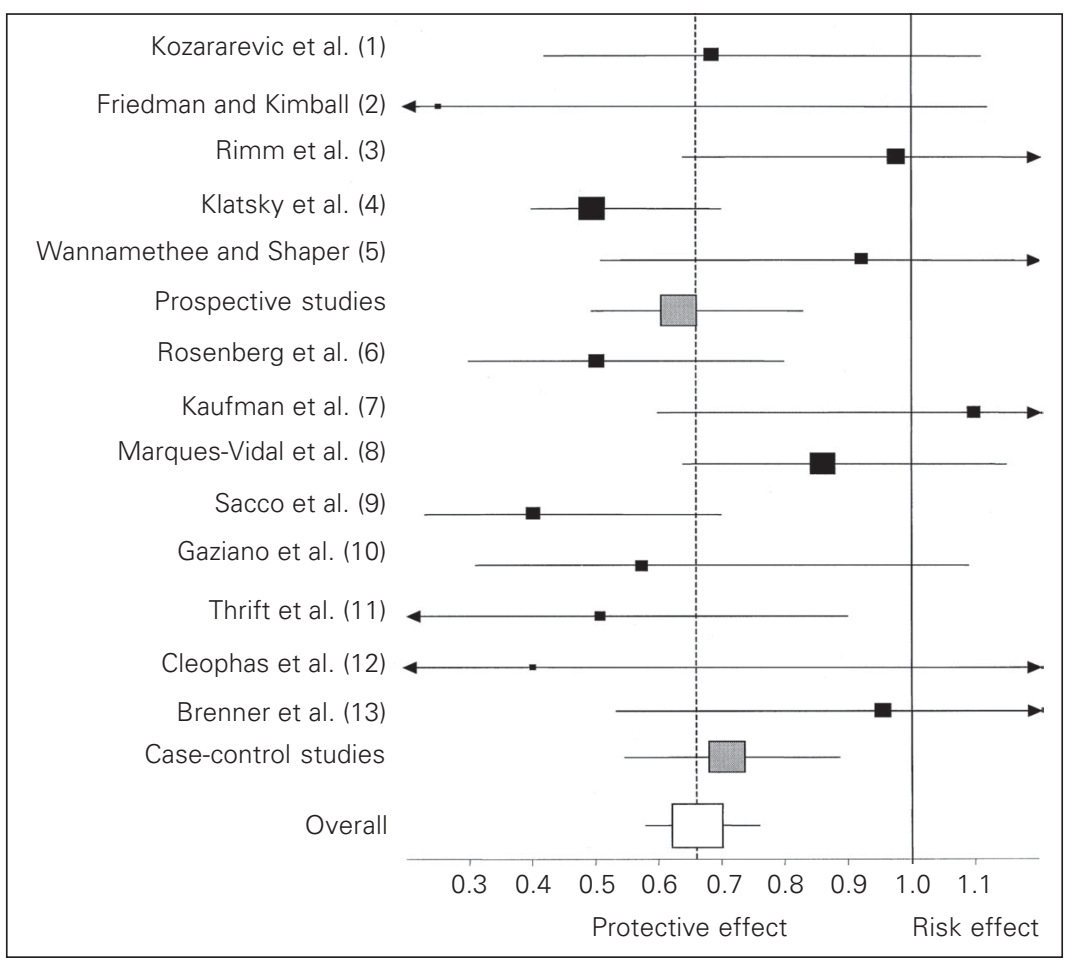

CAD compared to those who never or rarely consumed alcohol. Those two studies are noteworthy, among other reasons, because physicians are well educated and presumably trustful informers.

In the Nurses' Health Study (10), of 87,562 women ranging in age from 34 to 59 years and prospectively observed for 4 years, there was a significant decrease of CAD and ischemic stroke risk in individuals taking alcoholic beverages (one and a half dose a day with 10 to $15 \mathrm{~g}$ of alcohol) after adjustment for other risk factors. This suggests that moderate drinking may be beneficial for women also.

More recently, a meta-analysis (11) of 26 studies on wine or beer consumption and vascular risk was published (Figure 2). In 13 studies involving 209,148 wine-drinking persons the relative risk for vascular disease

Figure 2. Meta-analysis of wine and beer intake and vascular risk (see text). Modified from Ref. 11, with permission. (1) Kozararevic D, McGee D, Vojvodic N, Racic Z, Dawber T, Gordon T \& Zukel W (1980). Lancet, 1: 613-616. (2) Friedman LA \& Kimball AW (1986). American Journal of Epidemiology, 124: 481-489. (3) Rimm EB, Giovannucci EL, Willett WC, Colditz GA, Ascherio A, Rosner B \& Stampfer MJ (1991). Lancet, 338: 464-468. (4) Klatsky AL, Armstrong MA \& Friedman GD (1990). American Journal of Cardiology, 66: 1237-1242. (5) Wannamethee SG \& Shaper AG (1999). American Journal of Public Health, 89: 85-90. (6) Rosenberg L, Slone D, Shapiro S, Kaufman DW, Miettinen OS \& Stolley PD (1981). American Journal of Public Health, 71: 82-85. (7) Kaufman DW, Rosenberg L, Helmrich SP \& Shapiro S (1985). American Journal of Epidemiology, 121: 548-554. (8) Marques-Vidal P, Ducimetiere P, Evans A, Cambou JP \& Arveiler D (1996). American Journal of Epidemiology, 143: 1089-1093. (9) Sacco $R L$, Elkind M, Boden-Albala B, Lin IF, Kargman DE, Hauser WA, Shea S \& Paik MC (1999). Journal of the American Medical Association, 281: 53-60. (10) Gaziano JM, Hennekens CH, Godfried SL, Sesso HD, Glynn RJ, Breslow JL \& Buring JE (1999). American Journal of Cardiology, 83: 52-57. (11) Thrift AG, Donnan GA \& McNeil JJ (1999). Epidemiology, 10: 307-312. (12) Cleophas TJ, Tuinenberg E, van der Meulen J \& Zwinderman KH (1996). Angiology, 47: 789-796. (13) Brenner H, Rothenbacher D, Bode G, Marz W, Hoffmeister A \& Koenig W (2001). Epidemiology, 12: 390-395. 
was 0.68 compared to non-drinkers. A Jshaped curve was observed for the relation between wine and vascular risk. The relative risk for 208,036 beer drinkers, in 15 studies, was 0.78 ; however, there was no relation between the amount of beer and vascular risk. Hence, there was a significant inverse association between wine intake and vascular risk; but the relation with beer was difficult to interpret since no meaningful association with the amount of beer consumed was documented.

An angiographic study by Liu et al. (12) examined 323 men and 220 women who underwent coronary arteriography; among men, the number of severe stenoses was significantly decreased in all current drinkers compared to non-drinkers, but without a dose-response effect; among women no such relation was documented.

The hypothesis that a regimen containing wine and flavonoids could be beneficial to CAD patients was tested in the Lyon Heart Study (13), which evaluated whether or not a typical Mediterranean diet could decrease events after a first myocardial infarct. The Mediterranean diet, which included fish, red wine, fruits, and vegetables compared to the conventional prudent diet of the American Heart Association, significantly reduced the incidence of new coronary events by approximately $70 \%$, over a period of 4 years. Alpha-linoleic acid, which is a major component of the Mediterranean diet and is present specifically in olive oil, was significantly associated with an improved prognosis. Greater risk factors, such as hypercholesterolemia and hypertension were independent for event recurrence, indicating that the Mediterranean diet did not affect, at least qualitatively, the relationship between major risk factors and recurrence.

In summary, the data suggesting an inverse association between moderate alcohol consumption, especially red wine, and cardiac mortality are extensive and highly suggestive, but still not definitive especially because they are based mostly on observational research.

\section{Stroke}

The relationship between drinking and stroke as a whole is difficult to analyze on the basis of the available literature. Although the studies are numerous, they are heterogeneous regarding samples, methods of data analysis, general characteristics (retrospective, case control, cohort), and type of beverage, i.e., wine, beer or liquor.

Ischemic stroke may be due to atherosclerosis in the carotid or cerebral arteries, as a result of plaque formation and thrombi, usually in older individuals. In the Copenhagen City Heart Study (14), 13,329 men and women aged 45 to 84 years were followed for 16 years; after adjustment for age, sex and smoking habits, a lower risk for stroke was observed after moderate wine intake, which was not confirmed with the use of beer or other distilled beverages.

In the Bruneck Study (15), a case-control study, in 460 patients, regular moderate alcohol intake decreased the incidence of carotid stenosis; a J-shaped curve describing the relationship between stroke and alcohol consumption was observed with light drinkers facing a lower risk than abstainers or heavy drinkers; high doses of alcoholic beverages also increased gamma-glutamyl transferase, indicating hepatic lesion.

Considering prospective studies only, no consistent pattern can be identified. For instance, a Japanese study (16) analyzed the relationship between drinking habit surveyed in 1965 and cause-specific mortality over 19 years, in 5135 male Japanese physicians, taking into account smoking habit and separating ex-drinkers from non-drinkers. There was a weak, but significant, association between stroke and drinking, and this relation did not differ between hemorrhagic stroke and other types of stroke. The study also showed a significantly higher risk of total 
stroke and ischemic stroke for ex-drinkers, but not for occasional drinkers.

Klatsky et al. (17), in a prospective study of 123,840 persons with 1002 cardiovascular (600 CAD) deaths, showed that alcohol use was associated with a higher risk of mortality from hypertension, hemorrhagic stroke and cardiomyopathy, but with a lower risk from $\mathrm{CAD}$, occlusive stroke and nonspecific cardiovascular syndromes. In conclusion, there is some evidence of an increased risk for hemorrhagic stroke and of beneficial effects in light-to-moderate drinkers regarding ischemic stroke.

The Department of Health Hypertension Care Computing Project (18) reported a prospective study of 6369 hypertensive subjects (3161 men); the relative risks both for drinkers compared with non-drinkers and for level of alcohol consumption were calculated for mortality from CAD, stroke, non-circulatory, and all causes. At presentation, $76 \%$ of the men and $48 \%$ of the women reported recent alcohol consumption. Male drinkers had a reduced risk of mortality from stroke and possibly from CAD. Similar results were observed in women regarding stroke but not regarding CAD. In men, risk of stroke mortality was lowest at 1-10 units per week.

In a Chinese study (19) of 18,244 men aged 45-64 years followed from 1986 to 1989, those who consumed 1-14 drinks a week had a $19 \%$ reduction in overall mortality as compared to non-drinkers after adjustment for age, level of education, and cigarette smoking. This protective effect was not restricted to any specific type of alcoholic drink. Although light to moderate drinking (28 or fewer drinks per week) was associated with a $36 \%$ reduction in death from CAD, it had no effect on death from stroke, which is the leading cause of death in this population. As expected, heavy drinking (29 or more drinks per week) was significantly associated with increased risks of death from stroke.

A linear, but nonsignificant, relationship for all drinking categories was reported in two recent European studies (20,21). Maskarinec et al. (22) analyzed 5766 men aged 35-64 over 21 years of follow-up, in relation to alcohol units consumed per week. The risk for all cause mortality was similar for non-drinkers and men drinking up to 14 units a week. A strong positive relation was seen between alcohol consumption and risk of mortality from stroke, with men drinking 35 or more units having double the risk of non-drinkers, even after adjustment.

Hansagi et al. (23) suggested an elevated risk of death in male drinkers for both total stroke and ischemic stroke. Moreover, an increased risk was shown for men who reported occasional binge drinking and for those who reported occasional binges and intoxication. For women, ex-drinkers showed the highest risk of dying from both total and ischemic strokes, while weekly drinkers reported beneficial effects for both hemorrhagic and ischemic stroke. Beneficial effects were also found for those women who never indulged in alcohol binges and never felt intoxicated.

In summary, there is no clear evidence regarding the beneficial effect of light-tomoderate drinking on stroke occurrence as a whole. However, it is possible that the incidence of ischemic stroke is reduced while that of hemorrhagic stroke is not, the reason being that the main cause of hemorrhagic stroke is hypertension, and chronic heavy drinking causes hypertension. In addition, there is a lack of evidence regarding the specific potential protective effect of wine drinking. In fact, the underlying pattern of alcohol intake seems to be one of the major factors contributing to the occurrence of stroke, probably due to hypertension.

\section{Peripheral vascular disease}

Intermittent claudication or other manifestations of peripheral arterial insufficiency represent manifestations of obstructive atherosclerotic disease of femoral, popliteal or 
lower extremity arteries. Their pathophysiology is essentially similar to that of atherosclerosis in other territories. In fact treatment of dyslipidemia decreases angina and intermittent claudication as well.

In the Edinburgh Artery Study (24), 1592 men and women aged 55-74 years were studied. There was no association between ankle brachial pressure index (ABPI) and alcohol consumption in women, but, in men, increasing alcohol consumption was associated with a higher ABPI indicating less severe disease. This relationship was linear rather than U-shaped. In multiple regression analysis, after age adjustment the ABPI was related to wine consumption but not to beer or spirits in men. Upon adjustment for age and cumulative lifetime cigarette smoking, the association of wine consumption with the ABPI remained statistically significant. The cited authors concluded that, in males, greater alcohol consumption is related to a higher ABPI and that any protective effect of alcohol is related to wine consumption rather than to beer or spirits.

\section{Alcohol intake and non-cardiovascular diseases}

The relationship between alcohol drinking and non-cardiovascular disease has also been examined. For instance, age-related macular degeneration is an important cause of blindness after 65 years of age. There is an association between menopause, smoking habit, serum cholesterol level, and macular senile degeneration; the probable mechanism is the increase in platelet aggregation and serum fibrinogen which may promote thrombosis and vascular obstructions. A protective effect of wine on age-related macular degeneration was also observed in comparison to subjects who did not drink (Figure 3). Interestingly, only wine showed a negative association with age-related macular degeneration in this NHANES-1 study (25), probably because red wine has a greater content of flavonoids than other alcoholic beverages; the sample consisted of 3072 adults, 45 to 74 years of age, and this was a casecontrol study.

In the Bordeaux region, in a prospective study of 3777 community members, a negative association was observed between moderate wine intake and Alzheimer's disease in individuals older than 65 years (26). After 3 years, 2273 subjects not demented at baseline were still available for follow-up.

Dementia and Alzheimer's disease have been investigated as well. It has been postulated that oxidative stress may play a key role in dementia. This is substantiated by the recent discovery of the protective effect of wine. In wine, the flavonoids - powerful antioxidant substances also contained in tea, fruits and vegetables - have been thought to offer such protection.

Truelsen et al. (27) reported a study among participants in the third Copenhagen City Heart Study (1991 to 1994), aged 65 years or more, who where screened using the MiniMental State Examination and subsequently examined for dementia. Eighty-three subjects were diagnosed with dementia and the remaining 1626 non-demented subjects were included as controls. The two groups were compared with regard to alcohol intake and type of alcohol assessed 15 years before. Average weekly total alcohol intake had no significant effect on risk of dementia. Monthly and weekly intake of wine was significantly associated with a lower risk of dementia. For beer and spirits, only a monthly intake of beer was significantly associated

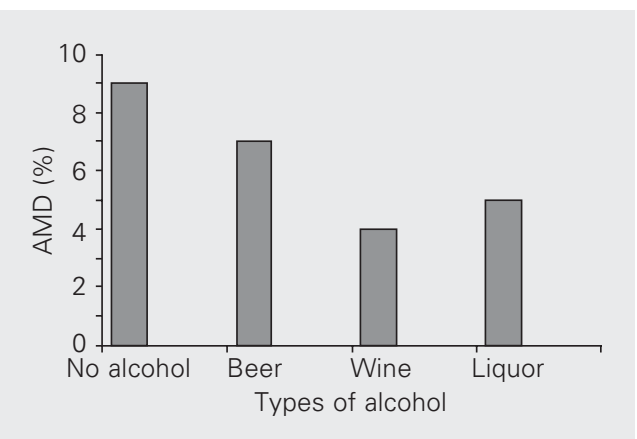

Figure 3. The protective effect of alcohol on age-related macular degeneration (AMD). Reproduced from Ref. 25, with permission. 
with an increased risk of dementia. The effect of alcohol on risk of dementia did not differ between men and women. Monthly and weekly intake of wine was associated with a lower risk of dementia. The results suggested that certain substances in wine may reduce the occurrence of dementia.

Commenges et al. (28) investigated whether or not flavonoid intake could be associated with a lower incidence of dementia in a cohort of 1367 subjects above 65 years of age. A questionnaire was used to evaluate their intake of flavonoids and subjects were followed up for 5 years between 1991 and 1996; 66 incident cases of dementia were observed. The age-adjusted relative risk of dementia was $0.55(\mathrm{P}=0.02)$. After additional adjustment for gender, education, weight, and vitamin $\mathrm{C}$ intake, the relative risk was $0.49(\mathrm{P}=0.04)$. The cited authors concluded that the intake of antioxidant flavonoids is inversely related to the risk of incident dementia.

Zuccala et al. (29) assessed the association between alcohol consumption and cognitive impairment in a series of older subjects enrolled in a multicenter pharmacoepidemiology survey. The association between average alcoholic intake and cognitive performance was assessed in 15,807 patients. Cognitive impairment was detected in 1693 (19\%) of 8755 drinkers and in 2008 (29\%) of 7052 nondrinkers $(\mathrm{P}<0.0001)$. After adjusting for potential confounders, alcohol consumption was associated with decreased probability of cognitive impairment (odds ratio, 0.75). The relationship between drinking level and cognitive dysfunction was nonlinear because the probability of cognitive impairment was decreased for moderate alcohol use as compared with abstinence, but was increased for daily consumption exceeding one wine-equivalent liter among men and 0.5 liter among women. This nonlinear association persisted when cerebrovascular and Alzheimer's disease were considered separately. The authors concluded that alco- hol abuse is associated with increased prevalence of cognitive dysfunction among older subjects; however, a daily alcohol consumption of less than $40 \mathrm{~g}$ for women and $80 \mathrm{~g}$ or less for men might be associated with a decreased probability of cognitive impairment.

Lindsay et al. (30) analyzed 6434 subjects aged 65 years or older, who were cognitively normal in 1991; 4615 were alive in 1996 and participated in the follow-up study. Their cognitive status was reassessed 5 years later by a similar two-phase procedure, including a screening interview, followed by a clinical examination when indicated. The analysis included 194 Alzheimer's disease cases and 3894 cognitively normal controls. Increasing age, fewer years of education, and the apolipoprotein $\mathrm{E}$ epsilon4 allele were significantly associated with increased risk for Alzheimer's disease. Use of nonsteroidal anti-inflammatory drugs, wine consumption, coffee consumption, and regular physical activity were associated with a reduced risk of Alzheimer's disease.

In a case-control study, Mukamal et al. (31) examined 373 cases with incident dementia and 373 controls aged 65 years and older. The controls were frequency-matched for age, death before 1999, and their attendance of a 1998-1999 clinic. Participants in this study underwent magnetic resonance imaging of the brain and cognitive testing between 1992 and 1994 and were followed up until 1999. Compared with abstinence, the adjusted odds for dementia was 0.65 among those whose weekly alcohol consumption was less than 1 drink, 0.46 for 1 to 6 drinks, 0.69 for 7 to 13 drinks, and 1.22 for 14 or more drinks ( $\mathrm{P}$ for quadratic term $=$ $0.001)$. A trend toward greater odds for dementia associated with heavier alcohol consumption was most apparent among men and participants with an apolipoprotein $\mathrm{E}$ epsilon4 allele. The authors found generally similar relationships of alcohol use with Alzheimer's disease and vascular dementia. 
Compared with abstinence, weekly consumption of 1 to 6 drinks was associated with a lower risk of incident dementia among older adults.

Thus, taken together, the above studies suggest some protection against mental degeneration by moderate drinking, although the mechanisms remain unclear.

Regarding diabetes mellitus, prospective studies have shown that moderate alcohol intake decreases the risk of mortality by $\mathrm{CAD}$ in diabetic and non-diabetic men as well. The results from recent studies on the association between alcohol consumption, insulin resistance and type 2 diabetes mellitus are very interesting. Insulin resistance is not only associated with type 2 diabetes, but also plays an important role in the pathogenesis of obesity and cardiovascular diseases. Flanagan et al. (32) performed intravenous glucose tolerance tests and compared the results to data on alcohol consumption in 154 subjects. They showed a positive correlation between insulin sensitivity and alcohol consumption in young adults, independent of gender. These data raise the intriguing possibility of a putative protective role of moderate alcohol consumption against the development of type 2 diabetes. Wei et al. (33) prospectively studied 8663 men whose fasting plasma glucose was measured. There were 149 incident cases of type 2 diabetes during 52,588 person-years of follow-up. The authors observed an elevated risk of developing type 2 diabetes in nondrinkers and men with high alcohol intake when compared with men who reported moderate alcohol intake. This would suggest that men with a high alcohol intake could possibly reduce their risk of developing type 2 diabetes if they could drink less. Also, prospective studies indicate that moderate alcohol intake decreases the risk of mortality by CAD in diabetic and non-diabetic men as well. In addition, Solomon et al. (34), during 39,092 person-years of follow-up for 15 years, showed that moderate alcohol consumption is associated with reduced CAD in women with type 2 diabetes mellitus. Valmadrid et al. (35) carried out a prospective cohort study on 983 older-onset diabetic individuals of both genders, mean age 68.6 years, during a mean follow-up of 12.3 years. Patients were interviewed about their past-year intake of alcoholic beverages. During the 3-year follow-up there was an overall beneficial effect of alcohol consumption in decreasing the risk of death due to CAD in people with older-onset diabetes.

The Hoorn Study (36), carried out on a cohort of 2393 subjects, 50 to 75 years of age, followed for 10 years, also demonstrated that moderate alcohol consumption was associated with a lower risk for mortality and diabetes.

Ajani et al. (37) examined the relation between light to moderate alcohol consumption and CAD in men with and without diabetes mellitus in a prospective cohort study involving 87,938 US physicians (2790 with diagnosed diabetes mellitus), who participated in the Physicians' Health Study. They were followed for an average of 5.5 years, and deaths with CAD as the underlying cause were counted. During 480,876 person-years of follow-up, 850 deaths caused by CAD were documented: 717 deaths among nondiabetic men and 133 deaths among diabetic men. The authors concluded that light to moderate alcohol consumption is associated with similar risk reductions in CAD among diabetic and non-diabetic men.

Thus, in general diabetics seem to benefit from moderate drinking, especially red wine. Of course, uncontrolled diabetics must refrain from alcoholic beverages.

A relationship of flavonoids with estrogen has also been reported. It has been suggested that resveratrol, present both in red wine and purple grape juice, may stimulate the estrogen agonist receptor in different degrees. Gehm et al. (38), based on its structural similarity to diethylstilbestrol, a synthetic estrogen, examined whether resvera- 
trol might be a phytoestrogen. At concentrations $(3-10 \mu \mathrm{M})$ comparable to those required for its other biological effects, resveratrol inhibited the binding of labeled estradiol to the estrogen receptor and activated transcription of estrogen-responsive reporter genes transfected into human breast cancer cells. This transcriptional activation was estrogen receptor-dependent, required an estrogen response element in the reporter gene, and was inhibited by specific estrogen antagonists. In some cell types (e.g., MCF-7 cells), resveratrol functioned as a superagonist (i.e., it produced a greater maximal transcriptional response than estradiol) whereas in others it produced activation equal to or less than that of estradiol. Resveratrol also increased the expression of native estrogen-regulated genes, and stimulated the proliferation of estrogen-dependent T47D breast cancer cells. The authors concluded that resveratrol is a phytoestrogen and that it exhibits variable degrees of estrogen receptor agonism in different test systems. Therefore, resveratrol may be considered a phytoestrogen (26). This could partially explain CAD prevention in women with a moderate wine intake, as observed in the Nurse's Study.

Investigators from Harvard (39) reported a protective effect of moderate alcohol consumption in the development of duodenal ulcer in a cohort of 47,806 men, 40-75 years of age, followed for an average of 6 years; the putative mechanism might be the reduction in the growth of the bacterium Helicobacter pylori.

Finally, according to a report by Platz et al. (40), moderate alcohol intake decreases the risk of developing benign prostatic hyperplasia (BPH). This study was based on data from 29,386 men, ranging in age from 40 to 75 years, who were followed up for 9 years; they were asked whether or not they had undergone surgery for prostatic enlargement. After controlling for age, race/ethnicity, body mass index, physical activity, and both for alcohol intake and smoking, there was a strong inverse association between moderate alcohol intake and total BPH (defined as surgery-defined cases or high-moderate/severe lower urinary tract symptom-defined cases), surgery for BPH, symptomatic BPH, and enlarged prostate and the association was attenuated at high alcohol intake. The authors also studied the influence of smoking and found that the elevated risk of $\mathrm{BPH}$ was largely confined to those who smoke; therefore, they concluded that moderate alcohol consumption and smoke cessation will reduce the risk of $\mathrm{BPH}$ and progression to surgery.

Briefly, many clinical evidences suggest that there is a protective effect of alcoholic beverages, especially red wine, on vascular diseases, as well as on some non-vascular diseases, namely Alzheimer and dementia, duodenal ulcer, diabetes mellitus, senile macular degeneration, and BPH. Although most of these studies were observational and the mechanisms of such beneficial effects are not yet clear, the consistency of the data strongly suggests that a protective effect of moderate drinking, especially red wine, may indeed exist.

\section{Experimental studies}

Experimental animal and in vitro studies with different models of atherosclerosis or its cellular determinants have confirmed the protective effect of wine or grape constituents. For example, Da Luz et al. (41), using Sudan IV staining for macroscopic identification of aortic atherosclerotic plaques as well as the intima/media ratio in rabbits fed a hypercholesterolemic diet for 3 months, showed that the area covered by atherosclerotic plaques was $69 \pm 9 \%$ in animals treated with diet alone, while it was significantly decreased to $38 \pm 9 \%$ in animals that received diet + red wine in their drinking water, and to $47 \pm 12 \%$ in those that received non-alcoholic wine products together with the fat diet. The intima/media ratio was also 
significantly reduced by both treatments in comparison to diet alone (Figures 4 and 5). Interestingly, these protective effects occurred despite persistence of very high total cholesterol and LDL, no changes in triglycerides and high-density lipoprotein (HDL) and no antioxidant effect as measured by the lag time of LDL oxidation. Red wine was slightly more effective than non-alcoholic wine product. The mechanisms of action of wine have not been clarified in this experiment.

Hayek et al. (42) showed that apolipoprotein E-deficient mice also had significantly reduced atherosclerotic lesion areas in the ascending aorta, when fed red wine, catechin or quercetin in comparison to placebo-treated mice. Accordingly, cellular uptake by macrophages of LDL derived from apolipoprotein E-deficient mice was also reduced. These findings were associated with reduced LDL susceptibility to oxidation after exposure to red wine or quercetin, and to a lesser extent to catechin.

Feng et al. (43) analyzed the effect of red wine and white wine on balloon injury to the abdominal aorta in rabbits fed a cholesterol diet for 6 weeks. Rabbits fed red wine had significantly less neointimal hyperplasia than controls while white wine had no significant effect. However, both red and white wine significantly reduced MCP-1 messenger ribonucleic acid (mRNA) and protein expression in the aorta. The authors attributed their findings to the antioxidant properties of red wine which have been shown to be greater than those of white wine.

Iijima et al. (44) extracted the total polyphenolic fraction of red wine and documented its potent inhibitory effect on the proliferation and DNA synthesis of cultured rat aortic SMCs. They also investigated its mechanisms, specifically the phases of cell cycle division. They found that red wine polyphenols decreased the binding of nuclear proteins to the activating transcription factor site in the cyclin A promoter gene and down- regulated the mRNA levels of transcription factors as well as cAMP-responsive element binding protein; thus, down-regulation of cyclin A gene expression may contribute to the antiproliferative effect of wine polyphenols.

Rosenkranz et al. (45) demonstrated that preincubation of vascular SMCs isolated from rat thoracic aorta with red wine, but not white wine, inhibited ligand binding and tyrosine phosphorylation of the platelet-derived growth factor $\beta$ receptor (PDGFR), which plays a critical role in the pathogenesis of atherosclerosis. They identified flavonoids of the catechin family as major components of red wine; they also observed that the concentrations of red wine/catechins that inhibited PDGFR in vitro correlated with serum levels after red wine consumption in humans.
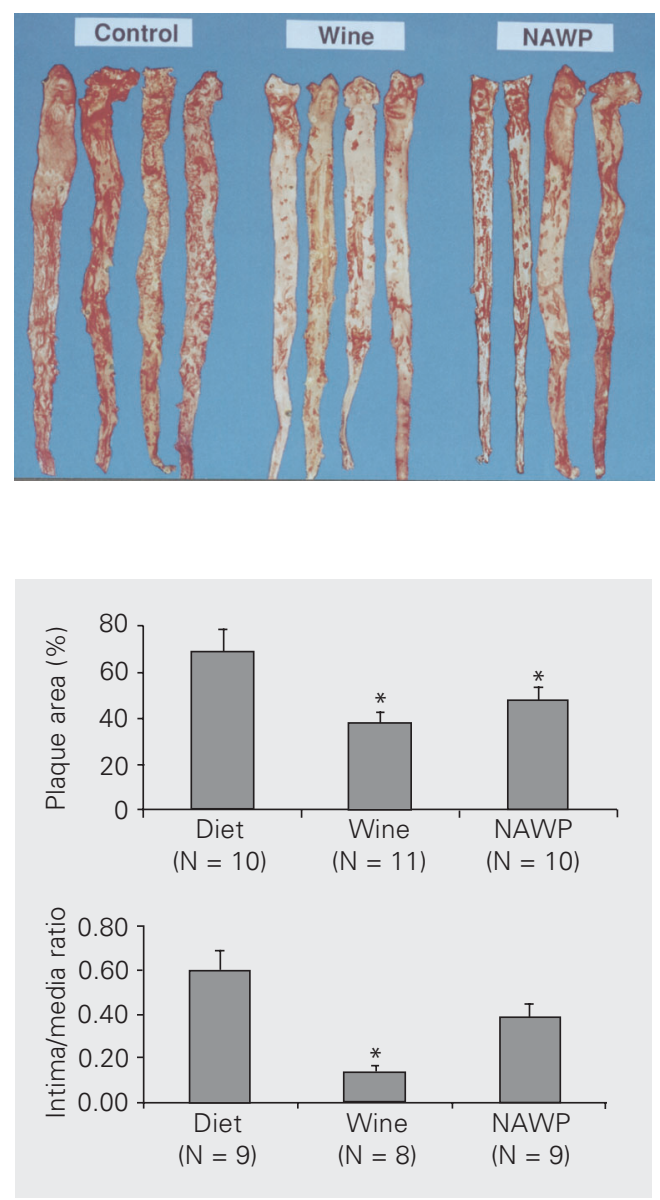

Figure 4. Examples of the effect of red wine and non-alcoholic wine products (NAWP) on macroscopic plaque formation in cholesterol-fed rabbits. Aortas were stained with Sudan IV and plaque area was measured by planimetry and reported as percent of total aortic surface. Red staining indicates atherosclerotic plaques. Control animals show more plaques than wine- or NAWP-treated rabbits. Reproduced from Ref. 41, with permission.

Figure 5. Plaque areas indicated by Sudan IV and intima/media ratio of the aorta measured microscopically. Data are reported as means \pm SEM. Both red wine (3.2 $\mathrm{ml} \mathrm{kg}^{-1}$ day $^{-1}$ ) and non-alcoholic wine products (NAWP) (3.2 $\mathrm{ml} \mathrm{kg}^{-1}$ day $^{-1}$ ) induced significant reductions in plaques and wine also significantly reduced intima/media ratio compared to diet controls. ${ }^{*} \mathrm{P}<0.05$ compared to diet (ANOVA). Reproduced from Ref. 41, with permission. 
Hence, several investigations indicate that red wine flavonoids may favorably interfere with key mechanisms of the atherosclerotic process, thus offering biological plausibility to the hypothesis of red wine/grape juice protection. It seems that most of the benefits accrued from alcoholic beverages can be attributed to these flavonoids.

\section{The J curve}

The relationship between mortality or cardiovascular events in humans and the intake of alcoholic beverages is consistently described by a $\mathbf{J}$ curve in essentially all studies. Therefore, abstainers and those who take more than $30 \mathrm{~g} /$ day of alcohol have increased overall mortality, higher blood pressure levels as well as increased hepatic enzymes; notably, heavy alcohol consumption sharply increases mortality in direct proportion to the dose. On the other hand, moderate alcohol intake (1-2 drinks/day) is associated with decreased CAD risk especially for mortality, hospitalization or angina, in both genders (46). Small daily doses seem to have a more protective effect than an equal single daily dose. Moderate doses of alcoholic beverages also have a preventive effect on individuals who already suffered a myocardial infarct, by reducing new coronary events and mortality. This J shape relationship has been observed regardless of type of beverage consumed.

Hypertension is an important risk factor for atherosclerosis, especially stroke, and is also influenced by alcohol. A J curve association between alcohol intake and blood pressure has been observed as well. The effect on blood pressure, with a low or moderate intake, is greater in women than in men.

Regarding sudden death and alcohol intake, studies by Albert et al. (9) have identified a U curve with a lower incidence after two to six doses of alcoholic beverage a day.

Therefore, those who do not take alcoholic beverages and those who take large quantities of alcohol have a greater risk for vascular events and adverse events whereas those who drink moderately have greater protection against vascular events.

\section{Adverse effects of alcohol}

Causes of increased mortality in heavy drinkers include cirrhosis, liver cancer, cancers of the upper aerodigestive tract (mouth, esophagus, larynx, and pharynx), accidents, suicide, homicide as well as cardiac events.

However, not only mortality should be considered as an undesirable side effect of alcohol consumption, but the various adverse effects of alcohol should never be underestimated. Alcohol consumption may cause gastrointestinal neoplasia, both fatal and nonfatal cardiac arrhythmias, automobile and industrial accidents, chronic alcoholism, psychosis, sexual harassment, and unemployment. The prevalence and severity of alcoholic cardiomyopathy are directly related to length of exposure to and the dose of alcohol. Alcohol has a negative inotropic effect that is dose dependent. In addition, alcoholism has been associated with reduced striated muscle strength as well as decreases in left ventricular ejection fraction.

In individuals with excessive alcohol intake it may be difficult to separate the effects of ethanol from socioeconomic influences, smoking, drug abuse, and type of diet. However, certain individuals surely should restrain from drinking, such as persons with a family history of alcoholism, elevated triglycerides, pancreatitis, liver disturbances, certain hematologic abnormalities, heart failure, uncontrolled arterial hypertension, pregnant women, and those using medications that may interfere with alcohol metabolism (sedatives, antidepressives).

\section{Mechanisms}

Several mechanisms may be involved in the effects of alcohol, especially red wine, 
such as actions on the endothelium, platelets, antioxidation, lipids, and cellular mechanisms.

\section{Flavonoids and endothelial function}

Several lines of evidence indicate that red wine or purple grape juice flavonoids promote endothelium-dependent dilation.

Red wine phenols block ET-1 production (47). Endothelin is a highly potent vasoconstrictor, induces SMC proliferation and its overproduction is a key factor in the development of atherosclerotic vascular diseases. Corder et al. (47) observed that polyphenols from different red wines decreased ET-1 synthesis by suppressing transcription of the ET-1 gen, in cultured bovine aortic endothelial cells. Red wine inhibition of ET-1 synthesis was directly correlated with total polyphenol content. White and rosé wines had no effect on ET-1 synthesis. Inhibitors of the cellular tyrosine-kinase family of phosphorylating enzymes that share structural similarity with red wine polyphenols also suppress ET-1 synthesis. The authors then investigated whether red wine polyphenols affected this family of enzymes, and indeed observed that in endothelial cells red wine caused a marked change in cell morphology and a redistribution of phosphotyrosine staining; they suggested that the effect of red wine was a modification of tyrosine-kinase signaling in endothelial cells.

In vitro, red wine and purple grape juice flavonoid components induce endotheliumdependent vasodilation of arterial rings (48), a phenomenon mediated by the NO guanosine cyclic monophosphate pathway (NO-cGMP).

In vitro the NO-cGMP pathway mediates the endothelium-dependent vasodilating effects of red wine and purple grape juice. Ethanol, at the same concentration as in red and white wines, does not cause vasorelaxation. Red wine or its extracts caused endothelium-dependent, NO-mediated vasorelaxation of rat (49) or rabbit aorta pre-con- stricted with norepinephrine (50). Also, in human coronary arteries and rat aortic rings in vitro, short-term incubation with red wine induced flow-mediated dilation and increased vascular cGMP content (51). The effects were abolished after endothelial denudation and reversed by endothelial nitric oxide synthase (eNOS) inhibition. Ethanol did not affect vascular tension or cGMP content.

Wallerath et al. (52) examined the molecular basis of the protective effect of wine. They treated human endothelial cells with red wine and measured eNOS mRNA expression, eNOS protein expression and eNOS activity and noted that incubation of endothelial cells with red wine upregulated eNOS mRNA and protein expression. Interestingly, these stimulatory effects of red wine were more pronounced with products originating from France than from Germany, and these effects did not differ between wines matured in oak barrels or steel tanks. Stimulation of the eNOS gene leads to enhanced NO production and presumably to its increased bioavailability. This up-regulation was seen in both EA.hy 926 cells and primary human umbilical vein endothelial cells; such phenomenon is mediated in part by increases in $\mathrm{Ca}^{2+}$ in endothelial cells.

In human studies, Stein et al. (53) analyzed the effects of purple grape juice ingestion in 15 individuals, mean age 62 years, with documented CAD, for 14 days. They observed significant improvement in flowmediated dilation and reduction in LDL susceptibility to oxidation. These effects were noted regardless of the use of antioxidant vitamins and lipid-lowering therapy. On the other hand, Hashimoto et al. (54) demonstrated that endothelium-dependent vasodilation improves after acute $(120 \mathrm{~min})$ intake of red wine or red wine without alcohol in men. But endothelium-independent vasodilation remained unchanged.

Djoussé et al. (55) studied 13 normal volunteers who were fed a high fat diet (0.8 $\mathrm{g} / \mathrm{kg}$ ) associated with red wine ingestion (3 
$\mathrm{ml} / \mathrm{kg}$ ). Their brachial artery flow-mediated dilation, measured by ultrasound, was not affected 2, 4 and $6 \mathrm{~h}$ after the meal. It should be noted that this was an acute study and not enough time may have elapsed to allow the effect of red wine to occur. In contrast, we examined endothelium-dependent brachial artery reactivity in 7 individuals with three coronary risk factors, namely, hypercholesterolemia, hypertension and smoking. Their baseline flow-mediated dilation was significantly reduced compared to values for normal individuals in our laboratory (5.7 vs $14.7 \%)$. After 7 days of red wine $(250 \mathrm{ml} /$ day) or purple grape juice (500 ml/day) consumption, flow-mediated dilation increased significantly at a similar proportion (Figure 6) (56). Interestingly, no significant changes occurred in plasma lipids, glucose or platelet aggregation. We also examined the effects of red wine and grape juice in 16 subjects of both sexes with isolated hypercholesterolemia (with no other risk factors); all subjects took red wine and grape juice in a random fashion for 14 days with an equal washout period between interventions. Again, we noted significant improvement, of equal magnitude, in flow-mediated dilation with both interventions without any significant change in plasma lipids, glucose or platelets, blood pressure or heart rate. In addition, grape juice but not red wine decreased ICAM1 significantly, but did not alter VCAM-1, indicating an anti-inflammatory action of grape juice. However, wine consumption also increased endothelium-independent dilation and the baseline brachial artery diam-

Figure 6. Red wine $(250 \mathrm{ml} /$ day) and grape juice $(500 \mathrm{ml} /$ day) for 7 days increase brachial artery flow-mediated dilation in 7 adult subjects of each group who had hypercholesterolemia, arterial hypertension and who smoked. ${ }^{*} P<0.05$ compared to before treatment days (ANOVA). GJ = grape juice; $\mathrm{RW}=$ red wine. $\mathrm{Re}$ produced from Ref. 56, with permission. eter. This fact can be explained by the para-

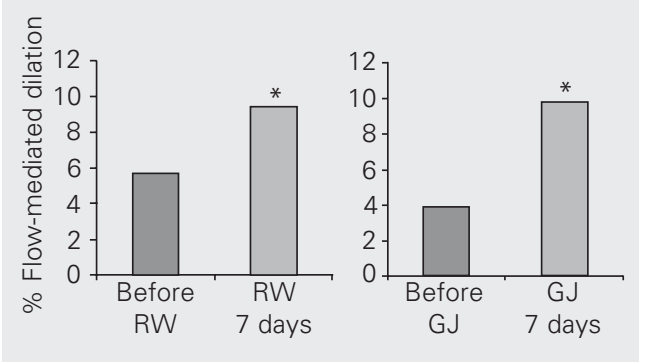

sympathetic nervous system activation and relative inactivation of the sympathetic nervous system which are known to occur after alcohol consumption. Another possible explanation is the effect of flavonoids and ethanol on NO production. Thus, Matsuo et al. (57) showed an increased acute production of NO with red wine and ethanol ingestion, but only a trend toward and increase in NO with polyphenols, in the plasma of 6 healthy subjects, using a new selective fluorescent indicator, diaminofluorescein, that allows direct detection of molecular NO under physiologic conditions. Note that the alcohol doses were the same in wine and alcohol itself, and polyphenols were given at the same concentration as in red wine. They also analyzed thiobarbituric acid-reactive substances as indicators of oxidative stress, and found no modifications. As far as we know, this is the first demonstration of acute NO production in humans stimulated by red wine and alcohol ingestion.

Teragawa et al. (58) also examined flowmediated dilation in 108 Japanese men with CAD; flow-mediated dilation was significantly increased in 54 men who drunk beer, sake, Japanese vodka, and whiskey at least once a week compared to those who did not drink. Note that in this Japanese study wine was not considered.

Hence, correction of endothelial dysfunction may be one mechanism by which red wine flavonoids protect against vascular disease.

\section{Antioxidation}

Increased resistance to LDL oxidation by flavonoids has been documented both experimentally and clinically. For instance, Stein et al. (53) gave $7.7 \mathrm{ml} \mathrm{kg}^{-1}$ day $^{-1}$ of purple grape juice, for 14 days, to 15 adults with angiographically documented CAD, and observed a $34.5 \%$ increase in the lag time to LDL copper-catalyzed oxidation, in addition to significant increases in flow-mediated di- 
lation. It is well known that oxidized LDL is toxic to endothelial cells and plays a significant role in the development and progression of atherosclerotic plaques. Flavonoids abundant in red wine and grape juice include resveratrol, quercetin, catechin, myricetin, kaempferol, and tannic acid. Quercetin is a powerful antioxidant and induces relaxation of aortic rings in vitro; catechins are also a family of powerful antioxidants which are present not only in red wine but similarly in vegetables, fruits, tea, and chocolate. In fact the consumption of large amounts of catechins may partially explain the protective effects of the Mediterranean diet, which is rich in fruits, vegetables and red wine. This is in accordance with the recent finding by Heitzer et al. (59) who analyzed forearm blood flow in 281 patients with CAD and related it to future cardiac events. They found that patients with cardiac events over a 4.5year follow-up had a lower vasodilatory response to acetylcholine but a greater benefit from local infusion of vitamin $\mathrm{C}$, thus indicating the importance of vessel oxidative stress. They concluded that endothelial dysfunction and vascular oxidative stress predicted the risk of future events in CAD patients.

Increased production of reactive oxygen species within the vessel has been recently considered an important mechanism for endothelial dysfunction. Specifically, superoxide reacts rapidly with $\mathrm{NO}$ and forms peroxynitrite anion causing loss of NO bioactivity. Reactive oxygen species, particularly peroxynitrite, can oxidize tetrahydrobiopterin, which is a critical co-factor for $\mathrm{NO}$ synthase. Thus, endothelial dysfunction can be at least partially reversed by different antioxidants. Hence, the antioxidant effect of flavonoids may be one mechanism by which red wine may protect against cardiac events.

\section{Platelet and hemostatic effects}

The effect of red wine and grape juice flavonoids on platelet function and hemostatic factors has also been studied (60). It was demonstrated that incubation of human blood with diluted purple grape juice caused a 3-fold increase in NO release from aggregating platelets. In addition, release of superoxide anion by aggregating platelets was reduced by $55 \%$. In humans, increased NO production and reduced superoxide release were also observed with grape juice ingestion. Reduced platelet aggregation was documented in vitro with both red wine and grape juice while orange juice and grapefruit had no significant effects. Similar results were observed in humans after a few weeks of daily moderate alcohol consumption. An inverse relation between fibrinogen levels and alcohol use has also been reported, as well as increased fibrinolytic activity with acute alcohol consumption. Platelet aggregation is a fundamental phenomenon in the development of atherosclerosis; in addition, platelets and fibrinogen, a fibrin precursor that is a major constituent of blood clots, are key participants in plaque instability. Conversely, the fibrinolytic system is of fundamental importance in clot destruction. Therefore, the effects described above may be important mechanisms of vascular protection by red wine and grape juice.

\section{Cellular effects}

As previously shown, important cellular effects of red wine or polyphenols have been documented. Thus, significant reductions in ICAM-1 were noted in human hypercholesterolemic subjects fed purple grape juice for 14 days (56), indicating an anti-inflammatory action of grape juice. NFkB production was significantly decreased by red wine ingestion in peripheral mononuclear cells of human volunteers fed a high fat breakfast; importantly, alcohol ingestion as vodka had no effect on NFKB production, indicating that the observed effect was related to flavonoids present in red wine, and not to alco- 
hol itself (61). Inhibition of PDGFR by red wine flavonoids was also observed in cultured vascular SMCs, an effect attributed mainly to catechins present in red wine flavonoids. Furthermore, blockade of SMC proliferation and cyclin A gene expression by red wine polyphenols was observed in vitro (45). MCP-1 mRNA and protein expression were reduced in the aorta of red wine-fed rabbits subjected to balloon injury (43).

Since all of these factors are key participants in the development, progression and instability of atherosclerotic plaques, their blockade or antagonism may represent an additional mechanism of vascular protection by red wine or grape juice flavonoids.

\section{Effects on lipids}

Increased HDL and triglycerides have been consistently documented in long-term alcohol use (60). For instance, Ruidavets et al. (62) carried out a cross-sectional survey of 1581 French men and 1535 French women, analyzing drinking habits and plasma lipids. The median daily alcohol intake was $24 \mathrm{~g}$ for men and $4 \mathrm{~g}$ for women. After adjustment for confounders, there was a positive and significant association between alcohol consumption and HDL and triglycerides in both sexes. Beer was also associated with HDL in men and with triglycerides in men and women. Considering drinking patterns, wine drinkers had higher HDL than non-wine drinkers. Since increases in HDL are known to exert a protective effect against events due to $\mathrm{CAD}$, this may also be a mechanism by which beverages may protect against atherosclerotic complications. On the other hand, no consistent changes have been documented regarding total cholesterol, LDL, VLDL, LP (a), or apolipoprotein B and E.

Psychological factors and higher social status also have been invoked as possible explanations for the health benefits of wine drinking. Thus, Mortensen et al. (63) analyzed 363 men and 330 women, aged 29 to
34 years, selected from the Copenhagen Perinatal Cohort. Socioeconomic status, education, IQ, personality, psychiatric symptoms, and health-related behaviors were considered in addition to wine or beer drinking habits. Wine drinking was significantly associated with higher parental educational level, higher socioeconomic status and even higher IQ, while beer drinking was associated with lower scores for those variables. The authors concluded that wine drinking was an indicator of optimal social, cognitive and personality development in Denmark, and speculated that these characteristics might explain the apparent benefit of wine drinking. It should be kept in mind, however, that this was a highly selected sample of young people; therefore, their findings may not be applicable to other populations, especially older ones.

\section{Conclusions}

Several lines of clinical evidence indicate a protective effect of moderate drinking on mortality and non-fatal events due to vascular diseases, specifically CAD. Data regarding cerebral vascular disease are inconsistent.

Experimental studies based on different models of atherosclerosis and focusing on chemical or cellular factors directly involved in the atherosclerotic process give support to the hypothesis that moderate drinking, especially of red wine, may indeed be protective. The mechanisms involved in the effects of red wine are principally related to flavonoids and include protection of endothelial function, antioxidation, antiplatelet effect, cellular mechanisms, and increases in HDL.

However, the relationship between alcohol consumption and clinical outcomes in humans has a J-shaped curve, indicating that the protective effect of beverages is only related to light/moderate drinking, i.e., 1-2 drinks/day (up to about $30 \mathrm{~g} / \mathrm{alcohol}$ ). Abstainers have no apparent benefit from their 
behavior, and heavy drinkers ( $\geq 60 \mathrm{~g}$ alcohol/ day) are clearly penalized by increases in mortality as well as the unacceptable consequences associated with alcoholism, such as accidents, gastrointestinal cancers or social disability. In addition, alcohol consumption is clearly contraindicated in certain types of patients such as those with hepatic disorders, cardiomyopathies, cardiac arrhythmias, or mental disorders. Furthermore, one must be aware of the potential problem of encouraging alcohol drinking in the young, since alcoholism is a major source of suicide and other undesirable behaviors in this group of individuals.
On the other hand, there seems to be no reason to prohibit moderate red wine drinking for people who are at risk of cardiovascular events and have no contraindications. Alternatively, purple grape juice may be recommended. By not being a restrictive measure, wine drinking becomes an attractive recommendation that may promote adherence of patients to medical prescription. Naturally, definite conclusions regarding ethanol-containing substances can only come from controlled, randomized, long-term clinical trials which seem exceedingly difficult to conduct since confounding factors are almost impossible to avoid.

\section{References}

1. Berliner JA, Navab M, Fogelman AM, Frank JS, Demer LL, Edward PA, Watson AD \& Lusis AJ (1995). Atherosclerosis: basic mechanisms. Oxidation, inflammation, and genetics. Circulation, 91: 24882496.

2. Steinberg D, Parthasarathy S, Carew TE, Khoo JC \& Witztum JL (1989). Beyond cholesterol. Modifications of low-density lipoprotein that increase its atherogenicity. New England Journal of Medicine, 320: 915-924.

3. Da Luz PL \& Uint L (2003). Endotélio na aterosclerose: interações celulares e vasomotricidade em endotélio e doenças cardiovasculares. In: da Luz PL, Chagas ACP \& Laurindo FR (Editors), Endotélio e Doenças Cardiovasculares. Atheneu, São Paulo, SP, Brazil.

4. Renaud S \& de Lorgeril M (1992). Wine, alcohol, platelets, and the French paradox for coronary heart disease. Lancet, 339: 1523-1526.

5. St Leger AS, Cochrane AL \& Moore F (1979). Factors associated with cardiac mortality in developed countries with particular reference to the consumption of wine. Lancet, 1: 1017-1020.

6. Gronbaek M, Deis A, Sorensen TI, Becker U, Schnohr P \& Jensen G (1995). Mortality associated with moderate intakes of wine, beer, or spirits. British Medical Journal, 310: 1165-1169.

7. Klatsky AL, Friedman GD \& Armstrong MA (1986). The relationships between alcoholic beverage use and other traits to blood pressure: a new Kaiser Permanent Study. Circulation, 73: 628-636.

8. Doll R, Peto R, Hall E, Wheatley K \& Gray R (1994). Mortality in relation to consumption of alcohol: 13 years' observations in male British doctors. British Medical Journal, 309: 911-918.

9. Albert CM, Manson JE, Cook NR, Ajani UA, Gaziano JM \& Hennekens $\mathrm{CH}$ (1999). Moderate alcohol consumption and the risk of sudden cardiac death among US male physicians. Circulation, 10: 944-950

10. Stampfer MJ, Colditz GA, Willet WC, Splizer FE \& Hennekens CH (1988). A prospective study of moderate alcohol consumption and the risk of coronary disease and stroke in women. New England Journal of Medicine, 31: 267-273.

11. Di Castelnuovo A, Rotondo S, lacoviello L, Donati MB \& Gaetano G
(2002). Meta-analysis of wine and beer consumption in relation to vascular risk. Circulation, 105: 2836-2844

12. Liu Y, Tanaka H, Sasazuki S et al. (2001). Alcohol consumption and severity of angiographically determined coronary artery disease in Japanese men and women. Atherosclerosis, 156: 177-183.

13. De Lorgeril M, Salen P, Martin JL, Monjaud I, Delaye J \& Manelle N (1999). Mediterranean diet, traditional risk factors, and the rate of cardiovascular complications after myocardial infarction. Circulation, 99: 779-785

14. Truelsen T, Gronbaek M, Schnohr P \& Boysen G (1998). Intake of beer, wine and spirits and risk of stroke: the Copenhagen City Heart Study. Stroke, 29: 2467-2472

15. Kiechl S, Willeit J, Egger G, Oberhollenzer M \& Aichner F (1994). Alcohol consumption and carotid atherosclerosis: evidence of dosedependent atherogenic and antiatherogenic effects. Results from the Bruneck Study. Stroke, 25: 1593-1598.

16. Kono S, Ikeda M, Tokudome S, Nishizumi M \& Kuratsune M (1986). Alcohol and mortality: a cohort study of male Japanese physicians. Journal of Epidemiology, 15: 527-532.

17. Klatsky AL, Armstrong MA \& Friedman GD (1990). Risk of cardiovascular mortality in alcohol drinkers, ex-drinkers and nondrinkers. American Journal of Cardiology, 66: 1237-1242.

18. Palmer AJ, Fletcher AE, Bulpitt CJ, Beevers DG, Coles EC, Ledingham JG, Petrie JC, Webster J \& Dollery CT (1995). Alcohol intake and cardiovascular mortality in hypertensive patients: report from the Department of Health Hypertension Care Computing Project. Journal of Hypertension, 13: 957-964.

19. Yuan JM, Ross RK, Gao YT, Henderson BE \& Yu MC (1997). Follow up study of moderate alcohol intake and mortality among middle aged men in Shanghai, China. British Medical Journal, 31: 18-23.

20. Hart CL, Smith GD, Hole DJ \& Hawthorme VM (1999). Alcohol consumption and mortality from all causes, coronary heart disease, and stroke: results from a prospective cohort study of Scottish men with 21 years of follow up. British Medical Journal, 318: 1725-1729.

21. Romelsjo A \& Eifman A (1999). Association between alcohol con- 
sumption and mortality, myocardial infarction, and stroke in 25 year follow up of 49,618 young Swedish men. British Medical Journal, 319: 821-822.

22. Maskarinec G, Meng L \& Kolonel LN (1998). Alcohol intake, body weight, and mortality in a multiethnic prospective cohort. Epidemiology, 9: 654-661.

23. Hansagi $H$, Romelsjo A, Gerhardsson de Verdier M, Andreasson S \& Leifmann A (1995). Alcohol consumption and stroke mortality; 20 year follow-up of 15,077 men and women. Stroke, 26: 1768-1773.

24. Jepson RG, Fowkes FG, Donnan PT \& Housley E (1995). Alcohol intake as a risk factor for peripheral arterial disease in the general population in the Edinburg Artery Study. European Journal of Epidemiology, 11: 9-14

25. Obisesan TO, Hirsch R, Kosoko O, Carlson L \& Parrott M (1998). Moderate wine consumption is associated with decreased odds of developing age-related macular degeneration in NHANES. Journal of the American Geriatrics Society, 46: 1-7.

26. Orgogozo JM, Dartigues JE, Lafont S, Letenneur L, Commenges D, Salamon R, Renaud S \& Breteler MB (1997). Wine consumption and dementia in the elderly: a prospective community study in the Bordeaux area. Revista de Neurologia, 153: 185-192.

27. Truelsen T, Thudium D \& Gronbaek M (2002). Copenhagen City Heart Study. Amount and type of alcohol and risk of dementia: the Copenhagen City Heart Study. Neurology, 59: 1313-1319.

28. Commenges D, Scoted V, Renaud S, Jacqmin-Gadda H, BargergerGateau P \& Gatigues JF (2000). Intake of flavonoids and risk of dementia. European Journal of Epidemiology, 16: 357-363.

29. Zuccala G, Onder G, Pedone C, Cesari M, Landi F, Bernabei R \& Cocchi A (2001). Gruppo Italiano di Farmacoepidemiologia nell' Anziano investigators. Dose-related impact of alcohol consumption on cognitive function in advanced age: results of a multicenter survey. Alcoholism, Clinical and Experimental Research, 25: 17431748.

30. Lindsay J, Laurin D, Verreault R, Hebert R, Helliwell B, Hill GB \& McDowell I (2002). Risk factors for Alzheimer's disease: a prospective analysis from the Canadian Study of Health and Aging. American Journal of Epidemiology, 156: 445-453.

31. Mukamal KJ, Kuller LH, Fitzpatrick AL, Longstreth Jr WT, Mittleman MA \& Siscovick DS (2003). Prospective study of alcohol consumption and risk of dementia in older adults. Journal of the American Medical Association, 289: 1405-1413.

32. Flanagan DE, Moore VM, Godsland IF, Cockington RA, Robinson JS \& Phillips KI (2000). Alcohol consumption and insulin resistance in young adults. European Journal of Clinical Investigation, 30: 297301.

33. Wei M, Gibbons LW, Mitchell TL, Kampert JB \& Blair SN (2000). Alcohol intake and incidence of type 2 diabetes in men. Diabetes Care, 23: 18-22.

34. Solomon CG, Hu FB, Stampfer MJ, Colditz GA, Speizer FE, Rimm EB, Willett WC \& Manson JE (2000). Moderate alcohol consumption and risk of coronary heart disease among women with type 2 diabetes mellitus. Circulation, 102: 487-488.

35. Valmadrid CT, Klein R, Moss SE, Klein BE \& Cruickshanks KJ (1999). Alcohol intake and the risk of coronary heart disease mortality in persons with older-onset diabetes mellitus. Journal of the American Medical Association, 282: 239-246.

36. De Vegt F, Dekker JM, Groeneveld WJ, Nijpels G, Stehouwer CD, Bouter LM \& Heine RJ (2002). Moderate alcohol consumption is associated with lower risk for incident diabetes and mortality: the Hoorn Study. Diabetes Research and Clinical Practice, 57: 53-60.

37. Ajani UA, Gaziano JM, Lotufo PA, Liu S, Hennekens $\mathrm{CH}$, Buring JE
\& Manson JE (2000). Alcohol consumption and risk of coronary heart disease by diabetes status. Circulation, 102: 500-505.

38. Gehm BD, McAndrews JM, Chien P \& Jameson JL (1997). Resveratrol, a polyphenolic compound found in grapes and wine, is an agonist for the estrogen receptor. Proceedings of the National Academy of Sciences, USA, 94: 14138-14143.

39. Aldoori WH, Giovannucci EL, Stampfer MJ, Rimm EB, Wing AL \& Willett WC (1997). A prospective study of alcohol, smoking, caffeine, and the risk of duodenal ulcer in men. Epidemiology, 8: 420424.

40. Platz EA, Rimm EB, Kawachi I, Colditz GA, Stampfer MJ, Willett WC \& Giovannucci E (1999). Alcohol consumption, cigarette smoking, and risk of benign prostatic hyperplasia. American Journal of Epidemiology, 149: 106-115.

41. Da Luz PL, Serrano Jr CV, Chacra AP, Monteiro HP, Yoshida VM, Furtado M, Ferreiro S, Gutierrez P \& Pileggi $F$ (1999). The effect of red wine on experimental atherosclerosis: lipid-independent protection. Experimental and Molecular Pathology, 65: 150-159.

42. Hayek T, Fuhrman B, Vaya J, Rosenblat M, Belinky P, Coleman R, Elis A \& Aviram M (1997). Reduced progression of atherosclerosis in apolipoprotein E-deficient mice following consumption of red wine, or its polyphenols quercetin or catechin, is associated with reduced susceptibility of LDL to oxidation and aggregation. Arteriosclerosis, Thrombosis, and Vascular Biology, 17: 2744-2752.

43. Feng AN, Chen YT, Chen BS, Ding YZ \& Lin SJ (1999). Red wine inhibits monocyte chemotactic protein-1 expression and modestly reduces neointimal hyperplasia after balloon injury in cholesterol fed rabbits. Circulation, 100: 2254-2259.

44. lijima K, Yoshizumi M, Hashimoto M et al. (2000). Red wine polyphenols inhibit proliferation of vascular smooth muscle cells and downregulate expression of cyclin A gene. Circulation, 101: 805-811.

45. Rosenkranz S, Knirel D, Dietrich H, Flesh M, Erdmann E \& Bohm M (2002). Inhibition of the PDGF receptor by red wine flavonoids provides a molecular explanation for the "French paradox". FASEB Journal, 16: 1958-1960.

46. Marmot M \& Brunner E (1991). Alcohol and cardiovascular disease: the status of the $U$ shaped curve. British Medical Journal, 303: 565568.

47. Corder R, Douthwaite JÁ, Lees DM, Khan NQ, Santos ACV, Wood EG \& Canies MJ (2001). Health: endothelin-I synthesis reduced by red wine. Nature, 414: 863-864.

48. Chen CK \& Pace-Asciak CR (1996). Vasorelaxing activity of resveratrol and quercetin in isolated rat aorta. General Pharmacology, 27: 363-366.

49. Andriambeloson E, Stoclet JC \& Andriantsitohaina R (1999). Mechanism of endothelial nitric oxide-dependent vasorelaxation induced by wine polyphenols in rat thoracic aorta. Journal of Cardiovascular Pharmacology, 33: 248-254.

50. Cishek MB, Galloway MT, Karim M, German JB \& Kappagoda CT (1997). Effect of red wine on endothelium-dependent relaxation in rabbits. Clinical Science, 93: 507-511.

51. Flesch M, Schwarz A \& Bohm M (1998). Effects of red and white wine on endothelium-dependent vasorelaxation of rat aorta and human coronary arteries. American Journal of Physiology, 275: H1183-H1190.

52. Wallerath T, Poleo D, Li H \& Förstermann U (2003). Red wine increases the expression of human endothelial nitric oxide synthase, a mechanism that may contribute to its beneficial cardiovascular effects. Journal of the American College of Cardiology, 41: 471-478.

53. Stein JH, Keevil JG, Wiebe DA, Aeschlimann S \& Foltz JD (1999) 
Purple grape juice improves endothelial function and reduces the susceptibility of LDL cholesterol to oxidation in patients with coronary artery disease. Circulation, 100: 1050-1055.

54. Hashimoto M, Kim S, Eto M et al. (2001). Effect of acute intake of red wine on flow-mediated vasodilatation of the brachial artery. American Journal of Cardiology, 88: 1457-1460.

55. Djoussé L, Ellison RC, McLennan CE, Cupples LA, Lipinska I, Tofleer $\mathrm{GH}$, Jokce N \& Vita JA (1999). Acute effects of a high-fat meal with and without red wine on endothelial function in healthy subjects. American Journal of Cardiology, 84: 660-664.

56. Coimbra SR, Lage SH, Yoshida W, Brandizzi L \& Da Luz PL (2002). Ação do vinho tinto e suco de uva sobre reatividade vascular, agregação plaquetária e lípides plasmáticos em pacientes hipercolesterolêmicos. Revista da Sociedade de Cardiologia do Estado de São Paulo, 12: 90 (Abstract).

57. Matsuo S, Nakamura Y, Takahashi M, Ouchi Y, Hosoda K, Nozawa $M \&$ Kinoshita M (2001). Effect of red wine and ethanol on production of nitric oxide in healthy subjects. American Journal of Cardiology, 87: 1029-1031.

58. Teragawa H, Fukuda Y, Matsuda K, Higashi Y, Yamagata T, Matsuura $\mathrm{H} \&$ Chayama K (2002). Effect of alcohol consumption on endothelial function in men with coronary artery disease. Atherosclerosis,
165: 145-152

59. Heitzer T, Schlinzig T, Krohn K, Meinertz T \& Munzel T (2001). Dysfunction, oxidative stress, and risk of cardiovascular events in patients with coronary artery disease. Circulation, 104: 2673-2678.

60. Rimm EB, Willians P, Fosher K, Criqui M \& Stampfer MJ (1999). Moderate alcohol intake and lower risk of coronary heart disease: meta-analysis of effects on lipids and hemostatic factors. British Medical Journal, 319: 1523-1528.

61. Blanco-Colio LM, Valderrama M, Alvarez-Sala LA, Bustos C, Ortego M, Hérnandez-Presa MA, Cancelas P, Gómez-Gerique J, Millán J \& Egido J (2000). Red wine intake prevents nuclear factor- $\kappa B$ activation in peripheral blood mononuclear cells of healthy volunteers during postprandial lipemia. Circulation, 102: 1020-1026.

62. Ruidavets JB, Ducimetiere P, Arveiler D, Amouyel P, Bingham A, Wagner A, Cottel D, Perret B \& Ferrieres J (2002). Types of alcoholic beverages and blood lipids in a French population. Journal of Epidemiology and Community Health, 56: 24-28.

63. Mortensen EL, Jensen HH, Sanders AS \& Reinich JM (2001). Better psychological functioning and higher social status may largely explain the apparent health benefits of wine. Archives of Internal Medicine, 161: 1844-1848 